\title{
Inflammation in Hepatocellular Carcinoma Patients Undergoing Hepatectomy: An Important Target for Ongoing Study
}

\section{Editorial Comment on "The Impact of Histological Liver Inflammation on Oncology and the Prognosis of Patients Undergoing Hepatectomy for Hepatocellular Carcinoma"}

\author{
June S. Peng, MD' ${ }^{1}$, and Niraj J. Gusani, MD, $\mathrm{MS}^{2}$ \\ ${ }^{1}$ Division of Surgical Oncology, Department of Surgery, Penn State College of Medicine, Hershey, PA; ${ }^{2}$ Section of \\ Surgical Oncology, Baptist MD Anderson Cancer Center, Jacksonville, FL
}

Miyata et al. ${ }^{1}$ present an institutional series of patients undergoing hepatectomy for hepatocellular carcinoma (HCC) and examined the impact of histologic evidence of liver inflammation in the resection specimen on oncologic outcomes.

The retrospective study included 500 consecutive patients who underwent curative intent treatment with upfront hepatectomy for HCC between 1994 and 2015. The median age was 67 years, 390 patients were male (78.0\%), and hepatitis $\mathrm{C}$ was the most common etiology for cirrhosis $(49.0 \%)$. Major hepatectomy, including at least three segments, was performed in $27.4 \%$ and Clavien-Dindo grade III and higher complications occurred in $18.6 \%$. Liver inflammation was graded according to the New Inuyama Classification $^{2}$ which incorporates both degree of fibrosis (scores ranging from $\mathrm{F} 0$ for no fibrosis to $\mathrm{F} 4$ for cirrhosis) and degree of necro-inflammatory reaction (scores ranging from A0 for no activity to A3 for severe activity). Severe (A3) liver inflammation was seen in $19.4 \%$ of patients, and histologic fibrosis classified as cirrhosis (F4) was seen in $35.8 \%$.

The authors found that severe liver inflammation was associated with worse RFS and OS. Unadjusted median recurrence-free survival for patients with A3 inflammation

(C) Society of Surgical Oncology 2022?

First Received: 3 January 2022

Accepted: 5 January 2022;

Published Online: 4 February 2022

N. J. Gusani, MD, MS

e-mail: niraj.gusani@bmcjax.com was 1.3 years compared with 3.3 years in those with A0-2 inflammation $(p<0.0001)$. Unadjusted median overall survival was 6.7 years for A3 compared with 10.4 years for A0-2 $(p=0.0013)$. Multivariate logistic regression for RFS identified multiple tumors (HR 2.17, 95\% CI 1.68-2.78), Child-Pugh class B (HR 2.02, 95\% CI 1.12-3.36), and A3 group (HR 1.36, 95\% CI 1.02-1.81) as risk factors for worse RFS. In the multivariate analysis, the A3 group was not independently associated with OS. The authors examined risk factors for early recurrence, defined as recurrence within 2 years of surgery, which occurred in $58.8 \%$ of the A3 group compared with $34.7 \%$ of the A0-2 group ( $p<$ $0.0001)$. Multivariate logistic regression identified multiple tumors (OR 2.44, 95\% CI 1.59-3.76), A3 group (OR 2.10, 95\% CI 1.25-3.55), and des-gamma-carboxy prothrombin $>40 \mathrm{mAU} / \mathrm{ml}$ (OR 1.59, OR 1.02-2.48) as risk factors for early recurrence.

Inflammation and the immune response play a vital role in cirrhosis, carcinogenesis, and cancer recurrence. Although the scoring system used by the authors for inflammation and fibrosis is not in widespread usage, the study results highlight the role of inflammation in development of cancers in cirrhosis and oncologic outcomes. Immunotherapy agents targeting PDL1, PD1, and CTLA4, are in various phases of investigation or approved for treatment of $\mathrm{HCC},{ }^{3}$ and the recent randomized IMbrave 150 trial demonstrated superiority of atezolizumab and bevacizumab over sorafenib for unresectable HCC (HR 0.58, 95\% CI 0.42-0.79). ${ }^{4}$

Although the role of intentional immunomodulation is not specifically addressed in this study, other groups have studied the impact of preoperative steroids in patients 
undergoing major hepatectomy. Bressan et al..$^{5}$ reported in a randomized, placebo-controlled trial that a single 500-mg dose of methylprednisolone prior to major hepatectomy resulted in shorter length of stay, fewer organ space infections, and lower bilirubin and international normalized ratio (INR) levels. A recent meta-analysis of randomized trials also demonstrated that steroid administration was associated with fewer postoperative complications, lower bilirubin, C-reactive protein (CRP), and interleukin-6 (IL6) levels. ${ }^{6}$ Multicenter trials are currently planned or underway. The authors of this study provide an important retrospective analysis of the link between liver inflammation and oncologic outcomes with thought-provoking correlations. However, caution should be taken to avoid inferring causality from a retrospective study. These results are intriguing but much study is needed to help elucidate the mechanisms and pathways involved. Management of pro-inflammatory insults should be optimized for patients with cirrhosis, with treatment for viral hepatitis or modification of risk factors. Prospective trials are needed to address the feasibility and benefit of immune modulation in this setting as well as how these manipulations may improve outcomes.

DISCLOSURES The authors have no relevant disclosures.

\section{REFERENCES}

1. Miyata T, Hayashi H, Yamashita Y-I, et al. The impact of histological liver inflammation on oncology and the prognosis of patients undergoing hepatectomy for hepatocellular carcinoma. Ann Surg Oncol. 2021. https://doi.org/10.1245/s10434-021-107067.

2. Ichida F, Omata M, Ichida T, et al. New Inuyama classification; new criteria for histological assessment of chronic hepatitis. Int Hepatol Commun. 1996;6(2):112-9.

3. Sangro B, Sarobe P, Hervás-Stubbs S, Melero I. Advances in immunotherapy for hepatocellular carcinoma. Nat Rev Gastroenterol Hepatol. 2021;18(8):525-43. https://doi.org/10.1038/s41575021-00438-0.

4. Finn RS, Qin S, Ikeda M, et al. Atezolizumab plus bevacizumab in unresectable hepatocellular carcinoma. $N$ Engl $J$ Med. 2020;382(20):1894-905. https://doi.org/10.1056/NEJMoa 1915745.

5. Bressan AK, Isherwood S, Bathe OF, Dixon E, Sutherland FR, Ball CG. Preoperative single-dose methylprednisolone prevents surgical site infections after major liver resection: a randomized controlled trial. Ann Surg. 2020;275(2):281-287. https://doi.org/ 10.1097/SLA.0000000000004720.

6. Hai HH, Aw P, Teng TZJ, Shelat VG. Perioperative steroid administration reduces overall complications in patients undergoing liver resection: a meta-analysis. World J Gastrointest Surg. 2021;13(9):1079-94. https://doi.org/10.4240/wjgs.v13.i9.1079.

Publisher's Note Springer Nature remains neutral with regard to jurisdictional claims in published maps and institutional affiliations. 